\title{
Projeto Terapêutico Singular no Âmbito da Saúde Mental: uma Experiência no Curso de Graduação em Medicina
}

\author{
A Unique Therapeutic Project in the Mental \\ Health Field: an Experience on an \\ Undergraduate Medical Course
}

Alcir Escocia Dorigatti ${ }^{I}$

Maria Laura Aguilar ${ }^{I}$

Ricardo M. Madureira

Felipe G. da Fonseca ${ }^{I}$

Rosana Teresa Onocko Campos ${ }^{I}$

Juliana Luporini Nascimento

\section{PALAVRAS-CHAVE}

- Medicalização;

- Saúde Mental;

- Psicotrópicos;

- Educação Médica.

\section{KEYWORDS}

- Medicalization;

- Mental Health;

- Psychotropic Drugs;

- Medical Education.

Recebido em: 08/04/2013

Aprovado em: 02/07/2013

\begin{abstract}
RESUMO
O Jardim Campo Belo, localizado no município de Campinas (SP), é um bairro de alta vulnerabilidade social e grande demanda por serviços de saúde, sendo a única oferta em saúde mental o matriciamento com outra unidade de saúde. Neste contexto, durante as disciplinas do segundo ano de Medicina, foi realizado um projeto de intervenção no Centro de Saúde, mediante acompanhamento em domicilio de pacientes usuários de psicotrópicos. Realizaram-se visitas quinzenais às casas de dez pacientes. Nestas visitas, trabalhou-se um roteiro semidirigido, em que se buscou conhecer as demandas de cada paciente. Alguns destes aumentaram os cuidados com a própria saúde, e num caso houve até suspensão do uso dos medicamentos psicotrópicos, em parceria com a equipe; em outros casos, as visitas serviram como válvula de escape social, tornando-se um espaço onde se ofertava atenção aos que estavam acostumados ao abandono social. Os alunos envolvidos relatam grande aprendizado graças às vivências e ao envolvimento com o tema, que se tornou um grande suplemento pedagógico.
\end{abstract}

\section{ABSTRACT}

Located in Campinas (SP). Jardim Campo Belo is a neighborhood characterized by high social vulnerability and a high demand for health services, with the only mental health service on offer accessible via another healthcare unit. It was in this context and during the second year of medical school, that we conducted an intervention initiative, monitoring patients using psychotropic drugs in their homes. Fortnightly visits were made to homes of 10 patients. During these visits, we conducted semi-directed interviews that allowed us to learn of the patients' needs. Some patients increased their own healthcare measures, in one case even being suspended from using psychotropic medications, in partnership with the health team. In other cases, visits served to provide a kind of sounding board, providing a moment for offering attention to individuals used to being neglected by society. Students involved reported having learned a great deal from the experiences and involvement with the subject, with it serving as a valuable educational supplement to the degree course. 


\section{INTRODUÇÃO}

O progresso curricular da educação médica nas Américas sofreu forte influência a partir de 1910, com a publicação do Relatório Flexner pelo educador norte-americano Abraham Flexner. ${ }^{1}$ No Brasil, as grandes alterações curriculares no curso de Medicina foram realizadas para atender a pressões internas e externas de instituições formadoras, seguindo as recomendações desse relatório. No entanto, a vigência das Diretrizes Curriculares Nacionais na década de 1990 trouxe questionamentos acerca dos currículos oferecidos até essa data, uma vez que foi proposta uma formação generalista, humanista, critico-reflexiva, baseada em princípios éticos².

A Faculdade de Ciências Médicas da Universidade Estadual de Campinas (FCM/Unicamp), acompanhando o movimento de reforma curricular das escolas médicas do País, implementou em 2001 a sua reforma, fruto de negociações e debates entre alunos, docentes e funcionários da faculdade. Nos moldes atuais, as disciplinas estão organizadas em Módulos de Ensino, e a estrutura curricular prioriza a inserção dos alunos de Medicina na rede municipal de saúde desde o primeiro ano do curso, buscando uma formação mais ética e com foco na responsabilidade social do profissional de saúde.

Nos dois primeiros anos, docentes do Departamento de Saúde Coletiva ministram dois Módulos: Ações em Saúde Pública I e II, e Saúde e Sociedade I e II, ambos anuais e com carga horária distribuída em atividades teóricas e de campo (práticas). Essas atividades são possíveis graças à consolidação de uma parceria com a Secretaria de Saúde da Prefeitura de Campinas e o Centro de Educação dos Trabalhadores da Saúde (CETS), que possibilitam a atuação dos alunos em Centros de Saúde de bairros carentes da cidade de Campinas. Durante dois anos, os alunos, divididos em pequenos grupos, trabalham numa mesma unidade de saúde, escolhida por eles no primeiro ano e com um mesmo tema, negociado com base em seus interesses e nos interesses e demandas do Centro de Saúde. Totalizam no primeiro e segundo ano do curso de graduação em Medicina nove Unidades Básicas da Rede de Saúde de Campinas como campos de estágio.

Inserido nessa realidade, este trabalho é fruto da experiência de alunos que realizaram sua formação nesses Módulos e com um tema específico. A Unidade Básica de Saúde (UBS) escolhida fica numa das regiões mais carentes da cidade, o Jardim Campo Belo, bairro da região sul de Campinas, que compreende uma população de baixa renda, num território marcado por alto índice migratório, sem rede de esgoto. Estudos demonstram a associação entre pobreza e saúde mental em países em desenvolvimento, indicando haver maior preva- lência de saúde mental precária em áreas geográficas também precárias, com populações de baixa renda.

Nesse contexto, o uso de psicotrópicos é bastante recorrente. Regiões de grande vulnerabilidade social, como o Campo Belo, possuem alta taxa de prescrição na Atenção Primária, e grande parte dos pacientes relata que os médicos tomam a decisão quanto ao tratamento a ser seguido com pouco ou nenhum grau de compartilhamento com os pacientes, considerando a terapêutica uma imposição em alguns casos, fator que limita a autonomia da população em questão e favorece o processo de medicalização do social. Essa tendência normativa da medicina sobre a sociedade passa a exercer um tipo de controle social que legitima o papel do doente ${ }^{3,4}$.

Além disso, 55\% dos pacientes de cinco UBS de Campinas usuários de benzodiazepínico afirmaram nunca terem recebido qualquer tipo de instrução quanto aos seus problemas de saúde, enquanto $22,5 \%$ receberam, mas não a entenderam. A prática generalista da prescrição de psicofármacos tem sido associada ao incremento da medicalização da população em detrimento da atenção às questões pessoais específicas de cada paciente. Qualquer sinal de sofrimento psíquico pode ser rotulado como uma patologia cujo tratamento consiste fundamentalmente na administração de psicofármacos, o que reforça o atual contexto de psiquiatrização da vida social ${ }^{4,5}$.

Dessa forma, observa-se que há quadros da saúde mental que não preenchem critérios diagnósticos e são iatrogenizados, além da possibilidade de uma submedicação daqueles com quadros expressivos, embora sejam situações mais raras. Ambas as situações poderiam ser evitadas com a melhoria da relação médico-paciente, incentivando a participação e cogestão no plano terapêutico. Isto se torna um grande desafio, principalmente porque devem se considerar o nível de escolaridade e a carência social da população-alvo, que pode sentir medo de questionar o único serviço onde consegue apoio. Neste aspecto, o Projeto Terapêutico Singular traz maior aproximação com estes pacientes, que, com a evolução do vínculo com o profissional, podem demonstrar aspectos antes não evidenciados numa consulta, muitas vezes tratada apenas como "renovação de receita" pelo prescritor.

\section{OBJETIVOS}

\section{Objetivo Geral}

O principal objetivo do projeto de intervenção foi atender à demanda por cuidado mais próximo e presente de parte dos pacientes em saúde mental do Jardim Campo Belo por meio da implantação de um Projeto Terapêutico Singular. O Jardim Campo Belo é uma região de alta vulnerabilidade social, sem 
atendimento de Centro de Apoio Psicossocial (Caps) e sem equipe de saúde mental na Unidade Básica de Saúde.

\section{Objetivos Específicos}

Formar um forte vínculo com a população atendida, possibilitando um meio de vazão para a pressão social que leva à medicalização das condições de carência de atenção resultantes da realidade social e da falta de suporte pela rede de serviços de saúde.

Compreender o modo como os pacientes entendem e administram o uso de medicamentos.

Possibilitar aos alunos o contato com os pacientes de saúde mental e permitir que aprendam como lidar com as demandas, estigmas e medo desses pacientes, tornando o projeto um instrumento pedagógico.

\section{METODOLOGIA}

Tratou-se de uma pesquisa qualitativa, em função da natureza do objetivo. $\mathrm{O}$ método utilizado foi o de pesquisa-ação, por meio de visitas reiteradas a um grupo de pacientes selecionados, com o propósito de construir para cada um deles um Projeto Terapêutico Singular.

O Projeto Terapêutico Singular (PTS) é um conjunto de propostas de condutas terapêuticas articuladas, para um sujeito individual ou coletivo, resultado da discussão de uma equipe interdisciplinar, com apoio matricial se necessário. Geralmente, é dedicado a situações mais complexas ${ }^{6}$.

Na elaboração do PTS, é preciso instituir o gerenciamento do caso, isto é, definir uma equipe ou um único profissional responsável pelas articulações necessárias à construção do PTS do portador. Este profissional discutirá com a equipe e familiares as necessidades dos pacientes, promoverá reuniões com a equipe e reavaliará as intervenções e as necessidades de atenção por meio de todos os serviços e instituições dentro da rede de saúde e fora dela ${ }^{7}$. Reconhece-se a capacidade gerencial do enfermeiro no cuidado dos pacientes devido a seu amplo conhecimento clínico, habilidade para oferecer cuidado holístico, advocacia a favor do paciente, conhecimento da rede de saúde e capacidade de otimização de recursos.

\section{Sujeitos}

O grupo foi construído por seleção proposital, ou seja, foram convidados intencionalmente, dentre os pacientes que fazem uso contínuo de medicação psicotrópica. Os pacientes que se enquadravam nesta situação foram encontrados através do livro de receitas controladas do Centro de Saúde Campo Belo.

O livro de receitas de medicação controlada contém todas as retiradas de medicamento que ocorrem no Centro de Saúde e registra o nome do paciente e o medicamento utilizado. Foram utilizados para compor a lista de pacientes todos aqueles que tinham seu nome no livro de receitas controladas no período de julho de 2009 a agosto de 2010. A lista foi composta com 69 pacientes.

Selecionaram-se os 20 maiores consumidores de medicamentos psicotrópicos por mais de seis meses (priorizando a quantidade de medicamentos diferentes) e buscaram-se os endereços de cada paciente nas pastas-prontuários do Centro de Saúde para início das atividades.

\section{Critérios de Inclusão}

- Ter mais de 18 anos de idade;

- Ser usuário de medicamento psicotrópico por mais de seis meses;

- Estar em condições físicas, emocionais e intelectuais minimamente satisfatórias para as visitas;

- Ter aceito a presença do aluno e a ida do aluno a sua casa com alguma frequência.

\section{Universo de Estudo}

No Centro de Saúde Campo Belo, não há equipe de Saúde Mental, porém os profissionais atuam no território através de matriciamento. O terapeuta ocupacional é proveniente do Centro de Saúde São Domingos, e o psiquiatra, do Centro de Saúde São José - situados próximos ao território do Centro de Saúde Campo Belo, o qual realiza apoio matricial. Semanalmente, programa-se uma agenda de triagem em Saúde Mental, que é realizada por um terapeuta ocupacional e uma enfermeira. Uma vez por semana, o psicólogo presta atendimentos na Unidade. $\mathrm{O}$ atendimento do psiquiatra é agendado no Centro de Saúde Campo Belo, porém os usuários são atendidos no Centro de Saúde São Domingos.

Os alunos envolvidos no projeto atuaram no campo em questão pelo período de dois anos, sendo que os seis primeiros meses foram de reconhecimento da realidade vivida pelos moradores, a fim de encontrar demandas onde pudessem intervir. Essa busca pelas necessidades dos moradores se deu através de visitas nas casas e conversas com os moradores do bairro.

Após o estudo de campo e a discussão das demandas, houve reunião com a equipe do Centro de Saúde Campo Belo, da qual todos participaram. Nessa reunião foram apresentadas as observações feitas ao longo do primeiro ano do ensino médico e proposta uma intervenção sob a forma de Projeto Terapêutico Singular no âmbito da saúde mental, por esta ter sido apontada como uma especialidade de alta demanda na região, porém com o cuidado pouco ofertado. 
Houve concordância da equipe do C. S. Campo Belo, e estabeleceu-se um vínculo de apoio, combinando-se que as agentes de saúde nos ajudariam a encontrar os pacientes e nos acompanhariam durante as atividades.

\section{Treinamento}

Todos os alunos envolvidos passaram por um treinamento antes de executarem as atividades, priorizando-se as habilidades de entrevista e o aprendizado de conceitos do campo da Psicologia médica. O material de estudo utilizado foi também ofertado à equipe do C. S. Campo Belo nas reuniões de evolução do projeto, realizadas no fim de cada semestre de 2010 e 2011.

\section{PTS - Etapa de Vínculo}

A viabilização da intervenção do PTS ocorreu primeiramente com o emprego da técnica da entrevista semiestruturada, iniciada com breve coleta de dados pessoais do informante, estabelecendo o rapport. Iniciada a entrevista com questões abertas, o pesquisador apresentava o tema da pesquisa por meio de uma questão disparadora. A partir daí, o entrevistado dirigia sua fala segundo livre associação de ideias. O entrevistador faz suas intervenções no discurso do sujeito para convidá-lo a aprofundar as ideias em exposição, assim como esclarecer pontos obscuros ao entendimento do ouvinte.

\section{PTS - Etapa de Acompanhamento}

Por meio do vínculo gerado na etapa anterior, seguiram-se atividades com o mesmo modelo. Porém, ao invés de usar uma questão disparadora, o entrevistador utilizava tópicos emergidos nas últimas conversas e que demonstravam as demandas e anseios do paciente, de forma a discutir e esclarecer dúvidas e estabelecer uma linha de trabalho. A cada encontro acontecia o rastreamento de novos temas, que foram sendo abordados enquanto se discutiram em profundidade alguns já apresentados pelo paciente.

\section{Discussão das Atividades e Replanejamento}

Como acontece no PTS, ao final de cada dia de atividade o que aconteceu semanalmente, respeitando o calendário da graduação - ocorria a discussão dos acontecimentos com o grupo, sob tutoria da docente responsável. Da mesma forma, discutia-se com a equipe, destacando-se as agentes de saúde, os próximos passos, os acertos e os erros do projeto. Estas agentes tiveram papel insubstituível para o surgimento do vínculo com os pacientes e para o progresso das atividades com suas sugestões.

Ao final de cada semestre foi realizada uma reunião com a equipe, quando também se discutiram melhoras e se refle- tiu em grupo sobre o que fazer com as informações obtidas e como dar prosseguimento às atividades.

\section{RESULTADOS E DISCUSSÃO}

\section{A Reforma Psiquiátrica}

A Reforma Psiquiátrica no Brasil é um movimento histórico de caráter político, social e econômico, influenciado pela ideologia de grupos dominantes. Foi proposta na década de 1980, sendo aprovada e colocada em prática apenas em 1999.

Uma de suas principais vertentes foi a desinstitucionalização, com a consequente desconstrução, do manicômio e dos paradigmas que o sustentam. A substituição progressiva dos manicômios por outras práticas terapêuticas e a cidadania do doente mental geraram muita polêmica quanto à eficiência das novas formas de tratamento e, especialmente, sobre esse novo olhar mais humano em relação à "loucura".

O modelo psiquiátrico proposto até então, baseado na legislação de 1934, propunha a hospitalização e o asilamento do doente mental, visando atender, sobretudo, à segurança da ordem e da moral pública. Embora tenha sido predominante, este modelo falido mostra uma base ideológica pejorativa, uma vez que a prioridade não era tratar o doente, mas proteger a sociedade dos possíveis perigos que ele poderia representar enquanto estivesse em liberdade ${ }^{8}$.

O novo modelo que vem sendo edificado com a reforma implica a desconstrução literal desse modelo hegemônico. O movimento aponta as inconveniências do modelo que fundamentou os paradigmas da Psiquiatria clássica e tornou o hospital psiquiátrico a única alternativa de tratamento, facilitando a cronicidade e a exclusão dos doentes mentais em todo o País. A Reforma Psiquiátrica, hoje defendida, é fruto de maior reflexão dos profissionais da saúde e da sociedade civil organizada, com base em conceitos teóricos e políticos da condução do programa psiquiátrico brasileiro ${ }^{9}$

Dados do Centro de Informações de Saúde, do Ministério da Saúde, revelaram que, em 1988, o número de leitos psiquiátricos representava 19,1\% do total de leitos disponíveis em todo o País, percentual superado apenas pelos leitos de Clínica Médica, $21,6 \%{ }^{10}$.

A análise do Gráfico 1 nos permite avaliar como a reforma implicou a redução dos leitos psiquiátricos em nosso país, revelando uma mudança de comportamento frente ao tratamento do doente mental. Nesse contexto, propõe-se que os Centros de Atenção Psicossocial (Caps) sejam responsáveis pela organização da rede de Saúde Mental, posicionando-se como uma referência para os demais serviços nas questões que envolvem sofrimento mental ${ }^{11}$. Tal posicionamento exige que esses serviços sigam uma clínica baseada no acolhimento 
e acompanhamento singular da construção subjetiva feita por cada paciente ${ }^{12}$. Esse trabalho exige a formação de vínculos que facilitem a aproximação entre profissionais de saúde, paciente, território e rede de saúde, de modo a proporcionar uma avaliação sensível e individualizada dos pacientes da saúde mental, analisando, para isso, o meio social que habitam ${ }^{13}$.

\section{GrÁfico 1}

Leitos psiquiátricos SUS por ano (2002-2006)

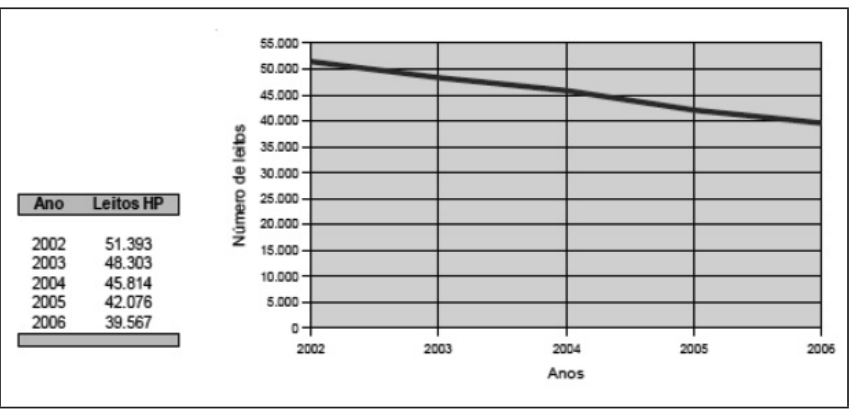

Fontes: Até o ano 2000, SIH/SUS. Em 2001, SIH/SUS, corrigido. Em 2002-2003, SIH/SUS, Coordenação Geral de Saúde Mental e Coordenações estaduais. Em 2004-2006, PRH/CNE

Apesar dos avanços trazidos pela Reforma Psiquiátrica para a saúde mental brasileira, ela criou um novo problema: deixamos de aprisionar os usuários em manicômios e hospitais psiquiátricos para aprisioná-los em suas casas, através de terapias medicamentosas sem prognóstico de fim, com a estigmatização que a doença mental traz perante a sociedade e com a medicalização social ${ }^{14}$.

Nesse sentido, é imprescindível a capacitação do profissional de referência, de tal modo que, mediante a elaboração compartilhada do projeto terapêutico, um profissional ou um grupo deles tomem para si os encargos do acompanhamento do paciente em suas diferentes facetas, tais como questões emocionais, familiares, educacionais, habitacionais, laborais e financeiras ${ }^{15}$. Cabe ao profissional de referência, em diálogo com a equipe técnica e o usuário, monitorar o projeto terapêutico individual, fazer contatos com a família do paciente e avaliar periodicamente as metas traçadas ${ }^{16}$.

\section{O Tratamento Medicamentoso}

Os estudos sobre medicamentos estão imbricados numa relação que envolve serviços de saúde e pacientes em determinados contextos sociais. Desse modo, o consumo de medicamentos é influenciado por concepções de seus consumidores e prescritores num diálogo contextualizado pelas condições socioeconômicas $^{17}$.
Compreendemos o termo "medicalização" como associado ao impacto da medicina ocidental na sociedade, em que está envolvida a popularização de terapias e de concepções biomédicas relacionadas ao processo saúde-doença. Tal percepção acerca do processo saúde-doença favorece o autoconsumo, fazendo dos medicamentos uma ferramenta para anular outros saberes e terapias não medicamentosas antes utilizadas como alternativa aos medicamentos ${ }^{18}$. A medicalização social é um fenômeno complexo, associado às transformações decorrentes da incorporação de condutas e modelos biomédicos na sociedade e a interpretações das experiências humanas exclusivamente segundo essas concepções. Associada à medicalização está a redução da perspectiva terapêutica, com desvalorização da abordagem de fatores sociais e subjetivos nos processos saúde-doença ${ }^{19}$.

No que se refere à prescrição de medicamentos, eles nem sempre são prescritos pelos serviços de saúde ou pelo médico, o que suporta a ideia de que o consumo de medicamentos participa de uma condição cíclica em que os consumidores também ditam como devem tomar, ora convencendo a prescrever, ora se automedicando, ora prescrevendo. Por isso, não reduzimos a medicalização à relação médico-paciente. $\mathrm{O}$ modo como esses medicamentos são usados nem sempre ocorre de acordo com as prescrições médicas, fazendo com que seu uso seja peculiar, de acordo com diferentes situações e condições socioeconômicas, e, muitas vezes, tornando a medicalização um processo social autônomo em relação às prescrições médicas, imersas nos fatores culturais da sociedade ocidental.

Os lares marcados por dificuldades econômicas e conflitos familiares, além de levarem ao consumo de calmantes, contribuem para definir o modo e quando serão consumidos. Os serviços de saúde, em função da alta demanda imposta à equipe e do baixo número de psiquiatras, acabam por não realizar as reavaliações periódicas do quadro clinico dos pacientes que fazem uso de psicotrópicos, caracterizando-se como fornecedores de prescrições medicamentosas.

Essa dependência medicamentosa gera anulação de respostas ao sofrimento social relacionado ao gênero, ao envelhecimento e às más condições econômicas e de qualidade de vida, promotoras de doenças psiquiátricas como ansiedade e depressão. Esse quadro pode ser intensificado nos idosos e ser estimulado por seu uso contínuo, principalmente no processo de envelhecimento da população brasileira mais carente ${ }^{20}$. Os efeitos indesejados dos calmantes são intensificados nos idosos, podendo levar a dependência, comprometimento cognitivo, sedação excessiva, tremores, quedas, lentidão psicomotora, diminuição da atenção e da memória. E, com seu uso crônico, esses efeitos podem silenciar doenças. Isto se acentua 
nas mulheres idosas: médicos podem prescrever medicamentos em decorrência da confusão entre aparência emotiva e sintomas de doenças mentais, como depressão e ansiedade, impondo e aceitando a mulher idosa como frágil ${ }^{21}$.

Neste contexto, a rede básica de atenção atua de forma a não incentivar a discussão e ofertas diversificadas para o usuário da saúde mental, perpetuando e aprisionando o tratamento numa prática estritamente farmacológica. Uma das saídas deste círculo vicioso é o emprego de Projetos Terapêuticos Singulares, como ocorreu no Centro de Saúde Campo Belo.

Muitos usuários deste estudo, ao menos seis deles, demonstraram não conhecer sua situação de saúde, estando aprisionados na prática de renovação de receitas sem reavaliações sistemáticas do quadro psiquiátrico; faltam a estes usuários espaços de reflexão sobre a própria condição, alcançada, em muitos casos, no acompanhamento junto aos postos de saúde. Esta conjectura é fruto, primeiramente, da condição de fragilidade em que o doente se encontra, não só pela doença como pelo estigma que ela traz consigo. Nesta situação, o médico aparece como grande apoiador psicológico e, ao optar pela terapia medicamentosa como primeira opção, fecha as demais para o paciente, tornando-o refém da medicação.

Houve casos de pacientes que se medicavam, concomitantemente, com nove medicamentos psicofarmacológicos, sem nunca terem sido consultados com um neurologista, mesmo apresentando um quadro epilético. Tais falhas podem ser resultados de falha no estabelecimento do vínculo na relação médico-paciente, muito facilitada pelo PTS, onde surgiram novas informações e possibilidades de ofertas.

Um caso de destaque no trabalho de campo se mostrou com uma senhora de 42 anos, usuária havia anos de três medicamentos. Após as primeiras visitas, ela se dispôs a compactuar com a equipe e buscar melhorar a qualidade de vida com caminhadas, dietas equilibradas e horários para dormir. Enquanto isso, ouviam-se as queixas da usuária em busca de formas para apoiá-la e promover novas ofertas. A paciente relatou melhora da saúde e demonstrou o desejo de abandonar os medicamentos, pois acreditava que estava se sentindo cada vez melhor. Sendo assim, orientamos que buscasse o serviço de matriciamento psiquiátrico do centro de saúde, e, com a avaliação do psiquiatra, ela deixou de usar os remédios, porém com visitas regulares de retorno. Ao fim do trabalho, emocionada, a paciente disse que era de um apoio próximo que ela estava precisando para melhorar. Note-se que, antes da intervenção do Projeto Terapêutico Singular, a paciente havia relatado desejo de se matar por envenenamento, pensamento de que hoje se arrepende.

\section{CONCLUSÕES}

A reorganização do cuidado em saúde mental, promovendo a des-hierarquização do atendimento em substituição por uma rede de serviços, trouxe a necessidade de revisar as antigas dinâmicas de acompanhamento prestadas. A visão do paciente internado ou ambulatorial cede lugar ao tratamento dos pacientes em seu próprio território.

Neste sentido, o Projeto Terapêutico Singular se consagra como uma forte estratégia na construção terapêutica destes pacientes, obtendo resultados expressivos como os descritos e muitas vezes com atitudes simples. O grande desafio está em permitir a aposta nesta forma de organização da assistência por toda a equipe, possibilitando ofertar ao paciente um momento de escuta e orientação terapêutica de acordo com suas próprias demandas, muitas vezes não observadas numa consulta de rotina.

\section{AGRADECIMENTOS}

A realização deste trabalho só foi possível graças ao apoio da equipe e funcionários do Centro de Saúde Campo Belo, no município de Campinas. Agradecemos a todos: diretora, médicos e equipes; principalmente às agentes de saúde, Cristina e Ivone, e ao psicólogo, Daniel, que nos trouxeram grandes ensinamentos com sua participação e por se prontificarem a nos ajudar.

\section{REFERÊNCIAS}

1. Pagliosa FL, Ros MAD. O Relatório Flexner: para o bem e para o mal. Rev. bras. educ. med. 2008;32(1):492-499.

2. Mourão LC, Martins RCB, Vieira CM, Rossin E, L'Abatte S. Análise Institucional e Educação: Reforma Curricular nas Universidades Pública e Privada. Educ. Soc. 2007; 28(98):181-210.

3. Santos DVD. Uso de psicotrópicos na Atenção Primária no Distrito Sudoeste de Campinas e sua relação com os arranjos da Clínica Ampliada: "Uma pedra no sapato". Campinas; 2009. Mestrado (Dissertação) — Universidade Estadual de Campinas.

4. Ferrazza DA, Luzio CA, Rocha LC, Sanches RR. A banalização da prescrição de psicofármacos em um ambulatório de saúde mental. Paidéia (Ribeirão Preto) 2010; 20(47)

5. Ribeiro CS, Azevedo RCS, Silva VF, Botega NJ. Chronic use of diazepam in primary healthcare centers: user profile and usage pattern. Sao Paulo Med J. 2007;6;125(5):270-4

6. Política Nacional de Humanização. Clínica ampliada, equipe de referência e projeto terapêutico singular. Ministério da Saúde, 2007. 
7. Monroe AA; Gonzales RIC; Sassaki CM; Netto AR; Villa TCS. Gerenciamento de caso ao doente/família com tuberculose: uma estratégia de sistematização do cuidado no domicílio. J. bras. Pneumol, v. 31, n.1, p. 91-92, 2005

8. Gonçalves AM, Sena RR. A Reforma Psiquiátrica no Brasil: Contextualização e Reflexos Sobre o Cuidado Com o Doente Mental na Família. Rev. Latino-am Enfermagem. 2001;9(2):48-55.

9. Amarante P. O Homem e a Serpente: Outras Histórias Para Loucura e a Psiquiatria. Rio de Janeiro: FIOCRUZ; 1996.

10. Alves DSN, Seidl EMF, Schechtman A, Silva RCl. Elementos Para Uma Análise da Assistência em Saúde Mental no Brasil. In: Conferência Nacional de Saúde Mental. A Reestruturaçäo da Atençäo em Saúde Mental no Brasil. Brasília: Ministério da Saúde; 1992. p. 46-59.

11. Miranda L, Onocko-Campos RT. Análise das Equipes de Referência em Saúde Mental: Uma Perspectiva de Gestão da Clínica. Cad. Saúde Pública. 2010; 26(6):1153-1162.

12. Tenório F. Questões para uma Atualização da Agenda da Reforma Psiquiátrica. In: Couto MCV, Martinez RG, org. Saúde Mental e Saúde Pública: Questões Para a Agenda da Reforma Psiquiátrica. Rio de Janeiro: Núcleo de Pesquisa em Políticas Públicas de Saúde Mental, Instituto de Psiquiatria, Universidade Federal do Rio de Janeiro; 2007. p. 11-28.

13. Leal EM, Delgado PGG. Clínica e Cotidiano. O Caps como Dispositivo de Desinstitucionalização. In: Pinheiro R, Guljor AP, Silva Jr. AG, Mattos RA, org. Desinstitucionalização da Saúde Mental: Contribuições para Estudos Avaliativos. Rio de Janeiro: Centro de Estudos e Pesquisa em Saúde Coletiva, Instituto de Medicina Social, Universidade do Estado do Rio de Janeiro/ABRASCO; 2007. p. 137-54.

14. Dorigatti AE, Belo KO, Kores RC, Trapé TL, Onocko Campos RT. El Usuario em Atención Básica y lãs ofertas em Salud Mental: La percepcíon de lós pacientes acerca de La medicación. Achivos de Medicina Familiar y General 2012;9(1):37-45.

15. Furtado JP. Equipes de Referência: Arranjo Institucional Para Potencializar a Colaboração Entre Disciplinas e Profissões. Interface (Botucatu). 2007; 11(22):239-55.

16. Miranda L, Campos RTO. Análise do Trabalho de Referência em Centros de Atenção Psicossocial. Rev. Saúde Pública. 2008;42(5):907-13.
17. Mendonça RT, Carvalho ACD, Vieira EM, Adorno RCF. Medicalização de mulheres idosas e interação com consumo de calmantes. Saúde Soc. 2008; 17(2):95-106.

18. Conrad P. Medicalization and Social Control. Annual Review of Sociology.1992;18:209-232.

19. Tesser CD, Barros NF. Medicalização social e medicina alternativa e complementar: pluralização terapêutica do Sistema Único de Saúde. Rev. Saúde Pública. 2008;42(5):914920.

20. Chaimowicz F, Ferreira TJXM, Miguel DFA. Uso de Medicamentos Psicoativos e seu Relacionamento com Quedas Entre Idosos. Rev. Saúde Pública. 2000;34(6):631-635.

21. Collin J. Médicament et vieillesse: trois cas de figure. Anthropologieet Sociétés;2003;27(2): 119-138.

\section{CONFLITO DE INTERESSES}

Declarou não haver.

\section{CONTRIBUIÇÃO DOS AUTORES}

Alcir Escocia Dorigatti: propôs o projeto, realizou o trabalho de campo, analisou e discutiu os dados e redigiu o artigo; Maria Laura Aguilar: realizou o trabalho de campo, analisou e discutiu os dados e colaborou na redação do artigo; Ricardo M. Madureira: realizou o trabalho de campo, analisou e discutiu os dados e colaborou na redação do artigo; Felipe G. da Fonseca: realizou o trabalho de campo, analisou e discutiu os dados e colaborou na redação do artigo;

Rosana Teresa Onocko Campos: realizou parte do trabalho de campo, orientando os alunos, participou da proposta inicial do projeto e trabalhou na elaboração final do manuscrito; Juliana Luporini Nascimento: realizou o trabalho de campo, orientando os alunos, analisou e discutiu os dados, validando os achados dos alunos, e colaborou na redação do artigo.

\section{ENDEREÇO PARA CORRESPONDÊNCIA}

Alcir Escocia Dorigatti

Rua Alexander Flemming, 181

Cidade Universitária Prof. Zeferino Vaz - Campinas

CEP 13083-887 — SP

E-mail: Alcir.dorigatti@gmail.com 